Proyecciones

Vol. 20, No 3, pp. 351-365, December 2001.

Universidad Católica del Norte

Antofagasta - Chile

\title{
NUMERICAL UNIFORMIZATION OF HYPERELLIPTIC-M-SYMMETRIC RIEMANN SURFACES *
}

\author{
RUBÉN HIDALGO \\ Universidad Técnica Federico Santa María, Chile
}

\begin{abstract}
In this note we consider hyperelliptic-M-symmetric Riemann surfaces, that is, hyperelliptic Riemann surfaces with a symmetry with maximal number of components of fixed points. These surfaces can be represented either by real algebraic curves or by real Schottky groups. To obtain one of these in terms of the other is difficult. In this note we proceed to describe explicit transcendental relations between the different sets of parameters these representations give. This can be used to obtain a computer program which permits obtain numerical approximations of the algebraic curve in terms of real Schottky group and viceversa.
\end{abstract}

Keywords : Schottky groups, Riemann surfaces, Riemann matrices.

*Partially supported by Projects Fondecyt 1000715, 7000715, 1010093 and UTFSM 12.01.22 


\section{Hyperelliptic-M-Symmetric Riemann Surfaces}

A closed Riemann surface $S$ of genus $g$ is called M-symmetric if it has a reflection $\tau: S \rightarrow S$ (that is, an anticonformal automorphism of order two with fixed points) with the maximal number of components of fixed points, that is $(g+1)$ componets. We also say that $\tau$ is a M-symmetry. If $g=0,1$, we say that $S$ is also a hyperelliptic-M-symmetric Riemann surface. For $g \geq 2$, we say that $S$ is hyperelliptic-M-symmetric if it is both M-symmetric and hyperelliptic. If $S$ is a hyperelliptic-M-symmteric Riemann surface $S$ of genus $g \geq 2$, with hyperelliptic involution $j: S \rightarrow S$ and M-symmetry $\tau: S \rightarrow S$, then the uniqueness of the hyperelliptic involution asserts that $j \tau=\tau j$. For genus $g=0,1$ this is not true due to the fact that hyperelliptic involution is not unique. Anyway, in that cases we may choose $j$ so that it commutes with $\tau$. Let us denote by $H$ the group generated by $j$ and $\tau$, then $H \cong \mathbf{Z} / 2 \mathbf{Z} \oplus \mathbf{Z} / 2 \mathbf{Z}$. The quotient surface $S / j$ is the Riemann sphere on which the $2(g+1)$ branch values of order 2 belongs to a common circle $L$, the fixed points of the induced reflection by $\tau$. The quotient $S / H$ is a closed disc with exactly $2(g+1)$ branch values of order 2 on its boundary. A hyperelliptic-M-symmetric Riemann surface $S$ can be described by an algebraic curve of the form $y^{2}=P(x)=x(x-1) \prod_{r=1}^{2 g-1}\left(x-a_{r}\right)$, where $1<a_{1}<a_{2}<\cdots<a_{2 g-1}<+\infty$. Also, this surface can be described by a certain real Schottky group (as described in the next section) depending on $2 g-1$ real numbers $1<\theta_{1}<\theta_{2}<\cdots<\theta_{2 g-1}<+\infty$. In this short note we find a relation between these two set of parameters corresponding to the same surface $S$. This relation is explicitly given and can be used to construct a computer program which permits to obtain one set of parameters from the other at least numerically (it means approximations). We must remark at this point that in [2] P. Buser and R. Silhol have considered this type of surfaces of genus two in order to find numerically an algebraic curve from a Fuchsian uniformization. We are replacing the Fuchsian group by a real Schottky group so his techniques does not work in this case. The idea is then to use Burnsides's arguments [1] in order to write down an explicit base of holomorphic one forms of the surface in terms of the uniformizing real Schottky group. 


\section{A Real Schottky Description of hyperelliptic- M-symmetric Riemann Surface}

Let us consider real numbers $1<\theta_{1}<\theta_{2}<\cdots<\theta_{2 g-1}<+\infty$ and set $\sigma_{1}$ be the reflection on the circle $C_{1}$, orthogonal to the real line through the points 1 and $\theta_{1}, \sigma_{k}$ be the reflection on the circle $C_{k}$, orthogonal to the real line through the points $\theta_{2 k-2}$ and $\theta_{2 k-1}$, for $k=2, \ldots, g$, and let $\sigma_{g+1}(z)=-\bar{z}$ be the reflection on the circle $C_{g+1}=\{i t: t \in \mathbf{R}\} \cup\{\infty\}$. Let us also consider $\sigma(z)=\bar{z}$ the reflection on the real line. The group $K$ generated by the reflections $\sigma$ and $\sigma_{k}, k=1, \ldots, g+1$, turns out to be a Kleinian group with connected region of discontinuity $\Omega$, keeping invariant the real line, with relations

$$
\sigma^{2}=\sigma_{k}^{2}=\left(\sigma \sigma_{k}\right)^{2}=1,
$$

and so that $\Omega / K$ is a closed disc with exactly $2(g+1)$ branch values of order two on its border (see figure 1).

Quasiconformal deformation theory and the fact that reflections has only circles has set of fixed points on the Riemann sphere assert that $S / H=\Omega / K$, for suitable values of $1<\theta_{1}<\theta_{2}<\cdots<\theta_{2 g-1}<$ $+\infty$.

Now, the index two subgroup $K^{+}$of $K$ consisting of the orientation preserving transformations turns out to be a Kleinian group (freely) generated by the involutions $L_{k}=\sigma_{k} \sigma, k=1, \ldots, g+1$. Each of these conformal involutions has both fixed points on the real line (see figure 2). We have that $\Omega / K^{+}$uniformizes the surface $S / j$. The index two subgroup $J$ of $K^{+}$generated by the hyperbolic transformations $A_{k}=L_{g+1} L_{k}=\sigma_{g+1} \sigma_{k}, k=1, \ldots, g$, turns out to be a real Schottky group, with associated reflection $\sigma$, so that $\Omega / G=S$ (see figure 3). We have that $J$ is a group of Möbius automorphisms of the hyperbolic plane $\mathbf{H}$. Let us consider the oriented loops $\alpha_{1}, \ldots, \alpha_{g}$, and the oriented paths $\beta_{1}, \ldots, \beta_{g}$, as shown in figure 4 . The projection of these oriented loops and arcs on the Riemann surface $S=\Omega / G$ is an adapted symplectic homology basis. The reflection $\tau: S \rightarrow S$, which is induced by $\sigma: \widehat{\mathbf{C}} \rightarrow \widehat{\mathbf{C}}$, in the above adapted symplectic homology basis, is represented by the following (extended) symplectic matrix

$$
\rho(\tau)=\left[\begin{array}{ll}
-I & 0 \\
0 & I
\end{array}\right]
$$


Since $\rho(\tau)(Z)=Z$, we have $-\bar{Z}=Z$ or equivalently

$$
\operatorname{Re}(Z)=0
$$

As a consequence of the results of Burnside [1], we get that the holomorphic differentials

$$
w_{k}(z)=\frac{1}{2 \pi i} \sum_{\gamma \in G} \frac{\gamma^{\prime}(z)}{\gamma(z)-A_{k}^{-1}(\infty)} d z, \quad k=1, \ldots, g,
$$

form a lifting of the dual holomorphic one-forms on $S$ respect to the (projected) oriented loops $\alpha_{1}, \ldots, \alpha_{g}$. The Riemann matrix of $S$, computed in the above adapted symplectic homology basis, is then

$$
Z=\left[\begin{array}{rrr}
z_{11} & \cdots & z_{1 g} \\
\vdots & \vdots & \vdots \\
z_{1 g} & \cdots & z_{g g}
\end{array}\right]
$$

where

$$
z_{k r}=\frac{i}{2 \pi} \log \left(\prod_{\gamma \in G} \frac{\left|\gamma\left(-q_{r}\right)-A_{k}^{-1}(\infty)\right|}{\left|\gamma\left(q_{r}\right)-A_{k}^{-1}(\infty)\right|}\right)
$$

and

$$
q_{r}= \begin{cases}1 & r=1 \\ \theta_{2 r-2} & 2 \leq r \leq g\end{cases}
$$

\subsection{An Algebraic Description}

The surface $S$ can be also represented by an algebraic curve of the form

$$
y^{2}=P(x)=x(x-1) \prod_{r=1}^{2 g-1}\left(x-a_{r}\right)
$$

where $1<a_{1}<a_{2}<\cdots<a_{2 g-1}<+\infty$. In this case the hyperelliptic involution $j$ and the reflection $\tau$ are given by:

$$
j=\left\{\begin{array}{l}
x \mapsto x \\
y \mapsto-y
\end{array}\right\} \quad \tau=\left\{\begin{array}{l}
x \mapsto \bar{x} \\
y \mapsto \bar{y}
\end{array}\right\}
$$

In [2] P. Buser and R. Silhol have considered this type of surfaces to determine (for genus two) a relation between the algebraic curve, 
the Fuchsian uniformization and Riemann matrices. We proceed in a similar fashion to obtain relations between the numbers $\theta_{1}, \ldots, \theta_{2 g-1}$ and the numbers $a_{1}, \ldots, a_{2 g-1}$, using the Riemann matrices. For this, first we know there is a conformal 1-1 mapping $f: D \rightarrow \mathbf{H}$, where $D$ denotes the common domain inside the hyperbolic plane $\mathbf{H}$ bounded by the circles $C_{1}, \ldots, C_{g+1}$. This map is unique if we ask the conditions $f(\infty)=-\infty, f(0)=0$ and $f(1)=1$. In this way, we have that $a_{k}=f\left(\theta_{k}\right)$, for $k=1, \ldots, 2 g-1$ (see figure 5 ). Using the principle of reflection, we can extend $f: D \rightarrow \mathbf{H}$ to a holomorphic branched covering $f: \Omega \rightarrow \widehat{\mathbf{C}}$ with $K^{+}$as covering group. The image under this branched covering map of the oriented loops $\alpha_{k}$ and paths $\beta_{k}, k=$ $1, \ldots, g$, is exactly the image under $\tau$ of the above projected homology basis on $S$. Let us denote by $\widehat{\alpha}_{k}$ the projection of $\alpha_{k}$ and by $\widehat{\beta}_{k}$ the projection of $\beta_{k}$ on the quotient $S / H$ (see figure 6 ).

Since the roots of $P(x)$ are given by $0,1, a_{1}, \ldots, a_{2 g-1}$, we may consider a square root $\sqrt{P(x)}$ in the simply connected region $\mathbf{C}-\left(L_{0} \cup\right.$ $\left.L_{1} \cup L_{a_{1}} \cup \cdots \cup L_{a_{2 g-1}}\right)$, where

$$
L_{k}=\{k+i y: y \leq 0\}
$$

For computations we must keep in mind the following facts:

(i) for $x<0$, we have $\sqrt{P(x)}=i \sqrt{|P(x)|}$;

(ii) for $0<x<1$, we have $\sqrt{P(x)}>0$;

(iii) for $1<x<a_{1}$, we have $\sqrt{P(x)}=-i \sqrt{|P(x)|}$;

(iv) for $a_{1}<x<a_{2}$, we have $\sqrt{P(x)}<0$;

(v) for $a_{2}<x<a_{3}$, we have $\sqrt{P(x)}=i \sqrt{|P(x)|}$; etc

A basis of holomorphic one-forms are given by

$$
\eta_{k}=\frac{x^{k-1} d x}{\sqrt{P(x)}}, \quad k=1, \ldots, g .
$$


Let us consider the (invertible) matrix

$$
A=\left[\begin{array}{lll}
\int_{\alpha_{1}} \eta_{1} & \cdots & \int_{\alpha_{g}} \eta_{1} \\
\vdots & \vdots & \vdots \\
\int_{\alpha_{1}} \eta_{g} & \cdots & \int_{\alpha_{g}} \eta_{g}
\end{array}\right]
$$

We have then that $W=A^{-1} E$, where

$$
W=\left[\begin{array}{l}
w_{1} \\
\vdots \\
w_{g}
\end{array}\right] \quad \text { and } \quad E=\left[\begin{array}{l}
\eta_{1} \\
\vdots \\
\eta_{g}
\end{array}\right]
$$

In particular, since we are using the same symplectic homology basis, we must have that

$$
Z=A^{-1} B
$$

where

$$
B=\left[\begin{array}{lll}
\int_{\beta_{1}} \eta_{1} & \cdots & \int_{\beta_{g}} \eta_{1} \\
\vdots & \vdots & \vdots \\
\int_{\beta_{1}} \eta_{g} & \cdots & \int_{\beta_{g}} \eta_{g}
\end{array}\right]
$$

\section{The Transcendental Relations}

The computations done in the two above sections give the same Riemann period matrix of the correspondient hyperelliptic-M-symmetric Riemann surface $S$. We are in the presence of two $(2 g-1)$-tupels:

$$
\left(\theta_{1}, \ldots, \theta_{2 g-1}\right) \text {; for the uniformizing Real Schottky group }
$$

and

$$
\left(a_{1}, \ldots, a_{2 g-1}\right) \text {; for the respective algebraic curve }
$$

To obtain relations between these two sets of parameters we observe that (because of the choice of the region on which we are computing the square root of $P(x)$ ) 


$$
\begin{gathered}
\int_{\alpha_{1}} \eta_{r}=2 \int_{\widehat{\alpha_{1}}} \eta_{r}=-2 \int_{1}^{a_{1}} \frac{x^{r-1} d x}{\sqrt{P(x)}}=-2 i \int_{1}^{a_{1}} \frac{x^{r-1} d x}{\sqrt{|P(x)|}} \in-i(0,+\infty) \\
\int_{\beta_{1}} \eta_{r}=2 \int_{\widehat{\beta_{1}}} \eta_{r}=2 \int_{0}^{1} \frac{x^{r-1} d x}{\sqrt{P(x)}} \in(0,+\infty)
\end{gathered}
$$

and for $k=2, \ldots, g$,

$$
\begin{gathered}
\int_{\alpha_{k}} \eta_{r}=2 \int_{\widehat{\alpha_{k}}} \eta_{r}=(-1)^{k} 2 i \int_{a_{2 k-2}}^{a_{2 k-1}} \frac{x^{r-1} d x}{\sqrt{|P(x)|}} \in(-1)^{k} i(0,+\infty) ; \\
k=2, \ldots, g
\end{gathered}
$$

and (by direct inspection of figure 6)

$$
\int_{\beta_{k}} \eta_{r}=2\left(\int_{\widehat{\beta_{1}}} \eta_{r}+\sum_{j=2}^{k-1}(-1)^{k} \int_{a_{2 j-3}}^{a_{2 j-2}} \frac{x^{r-1} d x}{\sqrt{P(x)}}\right) \in \mathbf{R}
$$

In this way, if we use the equality $B=A Z$, then we get explicit transcendental relations between the numbers $\theta_{1}, \ldots, \theta_{2 g-1}$ and the numbers $a_{1}, \ldots, a_{2 g-1}$.

\section{The classical Case: Genus One}

In the case of genus one Riemann surfaces the situation is very simple. In fact, each Riemann surface $S$ of genus one is represented by a parameter $\eta \in \mathbf{H}$. That is, $S=\mathbf{H} / \Gamma_{\eta}$, where $\Gamma_{\eta}$ is generated by the translations $A(z)=z+1$ and $B_{\eta}(z)=z+\eta$. A Schottky group $G_{\eta}$ uniformizing the same surface is generated by the loxodromic transformation $C_{\eta}(z)=e^{2 \pi \eta} z$. An algebraic curve $\mathcal{C}$ representing $S$ is given by

$$
y^{2}=4 x^{3}-g_{2}(\eta) x-g_{3}(\eta)
$$

where

$$
g_{2}(\eta)=60 \sum_{\gamma \in \Gamma_{\eta}-\{I\}} \frac{1}{\gamma(0)^{4}}
$$

and

$$
g_{3}(\eta)=140 \sum_{\gamma \in \Gamma_{\eta}-\{I\}} \frac{1}{\gamma(0)^{6}}
$$


As consequence of this note, we can also see this situation (for the case of genus one surfaces with a reflection with two components of fixed points) as follows. The algebraic curve of $S$ has the form

$$
y^{2}=x(x-1)(x-a)
$$

where $a>1$, and the real Schottky group depends on a real number $\theta>1$. In this case, we get the equality

$$
\frac{i}{2 \pi} \log \left(\prod_{k \in \mathbf{Z}} \frac{\left|A^{k}(-1)-\frac{\theta+1}{2}\right|}{\left|A^{k}(1)-\frac{\theta+1}{2}\right|}\right)=\frac{i \int_{0}^{1} \frac{d x}{\sqrt{x(x-1)(x-a)}}}{\int_{1}^{a} \frac{d x}{\sqrt{x(x-1)(a-x)}}}
$$

where

$$
A(z)=\frac{-(\theta+1) z+2 \theta}{2 z-(\theta+1)}
$$

Let us consider

$$
Q(z)=\frac{z-\sqrt{\theta}}{z+\sqrt{\theta}}=w
$$

and set

$$
\lambda_{\theta}=Q(-\theta)=\frac{1+\sqrt{\theta}}{\sqrt{\theta}-1}>1
$$

Then $Q(1)=\frac{-1}{\lambda_{\theta}}, Q(-1)=-\lambda_{\theta}$, and $Q(\theta)=\frac{1}{\lambda_{\theta}}$.

If we set $B(w)=Q\left(A\left(Q^{-1}(w)\right)\right)=\lambda_{\theta}^{2} w$, then the surface $S$ is also uniformized by the real Schottky group generated by $B$. It follows then that $S$ is uniformized by the group $F_{\theta}=\left\langle E(u)=u+1, F(u)=u+\eta_{\theta}\right\rangle$, where

$$
\eta_{\theta}=\frac{i \log \lambda_{\theta}}{\pi}
$$

and in particular,

$$
\frac{\log \lambda_{\theta}}{\pi}=\frac{1}{2 \pi} \log \left(\prod_{k \in \mathbf{Z}} \frac{\left|A^{k}(-1)-\frac{\theta+1}{2}\right|}{\left|A^{k}(1)-\frac{\theta+1}{2}\right|}\right)
$$

In this way, we have the most direct relation between $a$ and $\theta$ as

$$
\log \left(\frac{1+\sqrt{\theta}}{\sqrt{\theta}-1}\right)=\frac{\pi \int_{0}^{1} \frac{d x}{\sqrt{x(x-1)(x-a)}}}{\int_{1}^{a} \frac{d x}{\sqrt{x(x-1)(a-x)}}}
$$


Example. For instance, if we consider $a=2$, then the torus represented by the curve

$$
y^{2}=x(x-1)(x-2)
$$

admits an extra automorphism of order 4 , given by $A(x)=2 /(2-x)$. This corresponds to the parameter $\eta=i$ in the hyperbolic plane. The equality above then asserts that

$$
\theta \approx 1.18882 \ldots
$$

\section{Genus Two Case}

Let us consider a symmetric situation in order to make the computations more precise. For this we consider the one real parameter family of hyperelliptic-M-symmetric Riemann surfaces of genus 2 with an extra automorphism $W$ of order 3 which also commutes with the reflection $\tau$. These surfaces correspond to the algebraic curves defined by $a_{1}=\frac{a^{2}-a+1}{a}, a_{2}=a$ and $a_{3}=1-a+a^{2}$, for $a>1$, where $W(x)=\frac{x-a_{3}}{x-a_{2}}$. At the level of the real Schottky groups they correspond to have $\theta_{1}=\frac{1-\theta+\theta^{2}}{\theta}, \theta_{2}=\theta$ and $\theta_{3}=1-\theta+\theta^{2}$, for some value $\theta>1$. Similarly, the above automorphism $W$ of order 3 can be seen in the Schottky group as $W(z)=\left(z-\theta_{3}\right) /\left(z-\theta_{2}\right)$. The action of the automorphism $W$ at the level of the symplectic homology basis we have considered is given by

$$
W=\left[\begin{array}{rrrr}
0 & -1 & 0 & 0 \\
1 & -1 & 0 & 0 \\
0 & 0 & -1 & -1 \\
0 & 0 & 1 & 0
\end{array}\right]
$$

The Riemann period matrix $Z$ is then of the form

$$
Z=\frac{t i}{2}\left[\begin{array}{ll}
2 & 1 \\
1 & 2
\end{array}\right]
$$

for some $t>0$. From the real Schottky group we have the relation

$$
\frac{1}{2 \pi} \log \left(\prod_{\gamma \in G} \frac{\left|\gamma(-1)-\frac{\theta_{1}+1}{2}\right|}{\left|\gamma(1)-\frac{\theta_{1}+1}{2}\right|}\right)=t
$$


The relation with the parameter $a$ is given by

$$
t=-i \frac{\int_{\alpha_{2}} \eta_{2} \int_{\beta_{1}} \eta_{1}-\int_{\alpha_{2}} \eta_{1} \int_{\beta_{1}} \eta_{2}}{\int_{\alpha_{2}} \eta_{2} \int_{\alpha_{1}} \eta_{1}-\int_{\alpha_{2}} \eta_{1} \int_{\alpha_{1}} \eta_{2}}
$$

In particular, we obtain the relation

$$
\frac{1}{2 \pi} \log \left(\prod_{\gamma \in G} \frac{\left|\gamma(-1)-\frac{\theta_{1}+1}{2}\right|}{\left|\gamma(1)-\frac{\theta_{1}+1}{2}\right|}\right)=-i \frac{\int_{\alpha_{2}} \eta_{2} \int_{\beta_{1}} \eta_{1}-\int_{\alpha_{2}} \eta_{1} \int_{\beta_{1}} \eta_{2}}{\int_{\alpha_{2}} \eta_{2} \int_{\alpha_{1}} \eta_{1}-\int_{\alpha_{2}} \eta_{1} \int_{\alpha_{1}} \eta_{2}}
$$

Computationally, we evaluate the left hand side of the above equality just by using words of lenght at most certain $L>0$ in $G$. For the right hand side, there are numerical methods to obtain approximate values.

Example. Let us consider the following particular case. For $a=$ 2 , the above values $a_{1}=3 / 2, a_{2}=2$ and $a_{3}=3$, correspond to the hyperelliptic-M-symmetric closed Riemann surface of genus two admitting an extra automorphisms of order 6 (this is also described by the algebraic curve $\left.y^{2}=x^{6}-1\right)$. The automorphism of order 6 is given by $T(x)=3 /(3-x)$ and its square is $W$. At the level of the real Schottky group, this corresponds to some value $\theta_{1}=\frac{1-\theta+\theta^{2}}{\theta}, \theta_{2}=\theta$ and $\theta_{3}=1-\theta+\theta^{2}$, for some value $\theta>1$.

The matrices $A, B$ and $Z$ in this case give (numerical approximations):

$$
\begin{gathered}
A=\left[\begin{array}{rr}
-2.52083 i & 1.84538 i \\
-3.19628 i & 4.3662 i
\end{array}\right] \\
B=\left[\begin{array}{rr}
1.84537 & -0.675454 \\
1.16992 & -3.19628
\end{array}\right] \\
Z=A^{-1} B=\left[\begin{array}{rr}
1.1547 i & 0.57735 i \\
0.57735 i & 1.1547 i
\end{array}\right]
\end{gathered}
$$

This Riemann period matrix is known to be exactly

$$
Z=\frac{i}{\sqrt{3}}\left[\begin{array}{ll}
2 & 1 \\
1 & 2
\end{array}\right]
$$


that is, $t=\frac{2}{\sqrt{3}}$. Now, to obtain the value (numerical approximation) of $\theta$ we need to consider the equation

$$
\frac{1}{2 \pi} \log \left(\prod_{\gamma \in G} \frac{\left|\gamma(-1)-A_{1}^{-1}(\infty)\right|}{\left|\gamma(1)-A_{1}^{-1}(\infty)\right|}\right)=1.1547 \ldots=\frac{2}{\sqrt{3}}
$$

For this, we proceed to compute the left hand for words of $G$ of lenght less than certain $L>0$. In this way, we obtain an equation on $\theta$ to be pluged into Mathematica. For instance, using $L=3$ we obtain that $\theta \approx 1.87 \ldots$

\section{Ending Remark.}

The above two examples can be generalized for every genus $g \geq 3$. In fact, we use one real parameters of surfaces which are hyperellipticM-symmetric Riemann surfaces of genus $g$ which admit an extra automorphism $W$ of order $g+1$ which commutes with the reflection $\tau$. These families are codified by a parameter $a>1$ in the sense of the algebraic curves and by a parameter $\theta>1$ in the sense of real Schottky groups. These families contains a unique parameter $a$ and $\theta$ corresponding to the Riemann surface with an square root automorphism of $W$; such a surface is described also by the algebraic curve $y^{2}=x^{2(g+1)}-1$. Then, using the above equalities, we are able to find (in a similar way) numerical approximations of the values of $a$ and $\theta$ corresponding to such a surface. In this case, we have that the automorphism $W$ of order $g+1$ at the level of the algebraic curve is given by

$$
W=\frac{1}{\sqrt{\left(1-a_{1}\right)\left(1-a_{2}\right)\left(a_{2}-a_{1}\right)}}\left[\begin{array}{rr}
a_{2}-a_{1} & a_{1}(1-a 2) \\
a_{2}-a_{1} & 1-a_{2}
\end{array}\right]
$$

where

$$
a_{1}=\frac{4\left(1-a_{2}\right) a_{2} \cos ^{2}\left(\frac{\pi}{g+1}\right)-1}{4\left(1-a_{2}\right) \cos ^{2}\left(\frac{\pi}{g+1}\right)-1}
$$

The case $a_{1}=2-\frac{1}{a_{2}}$ corresponds to the symmetric case

$$
y^{2}=x^{2 g+2}-1
$$


At the level of he real Schottky group the form of $W$ is equivalent just by replacing the letter " $a$ " by the letter " $\theta$ " in the above formulae.

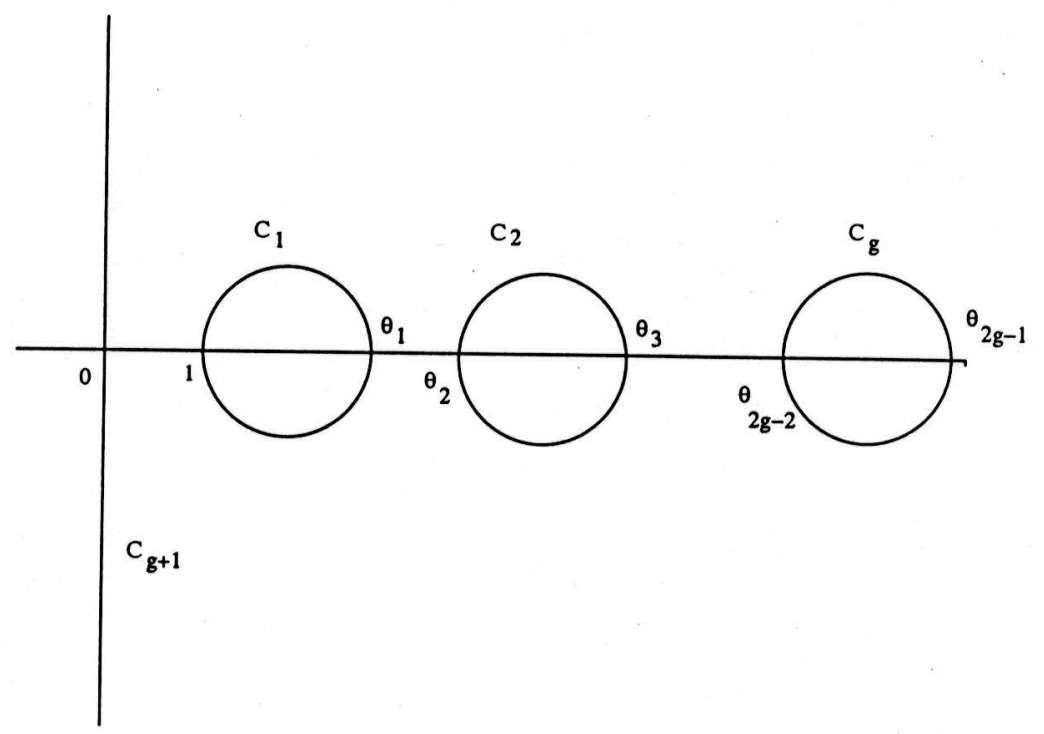



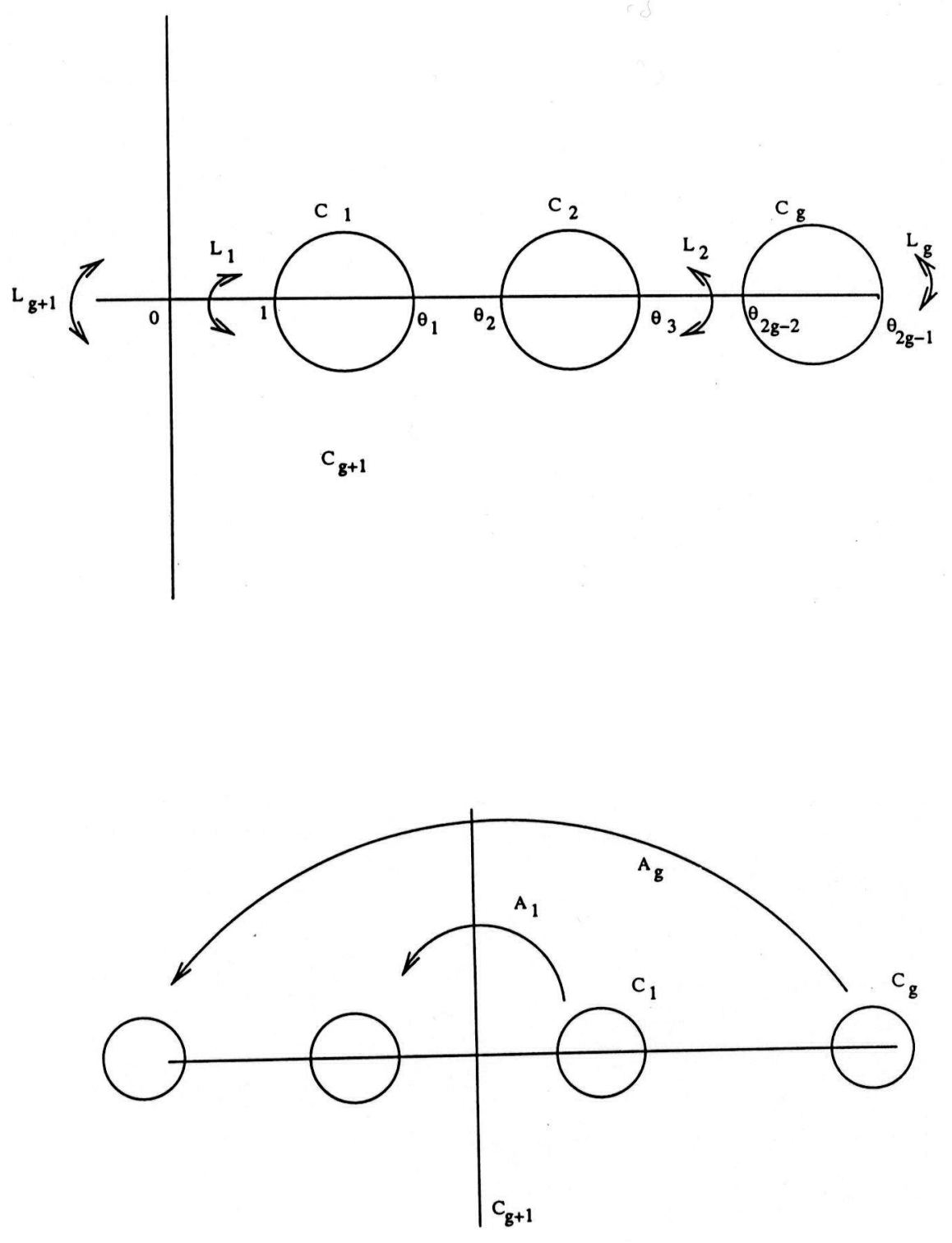

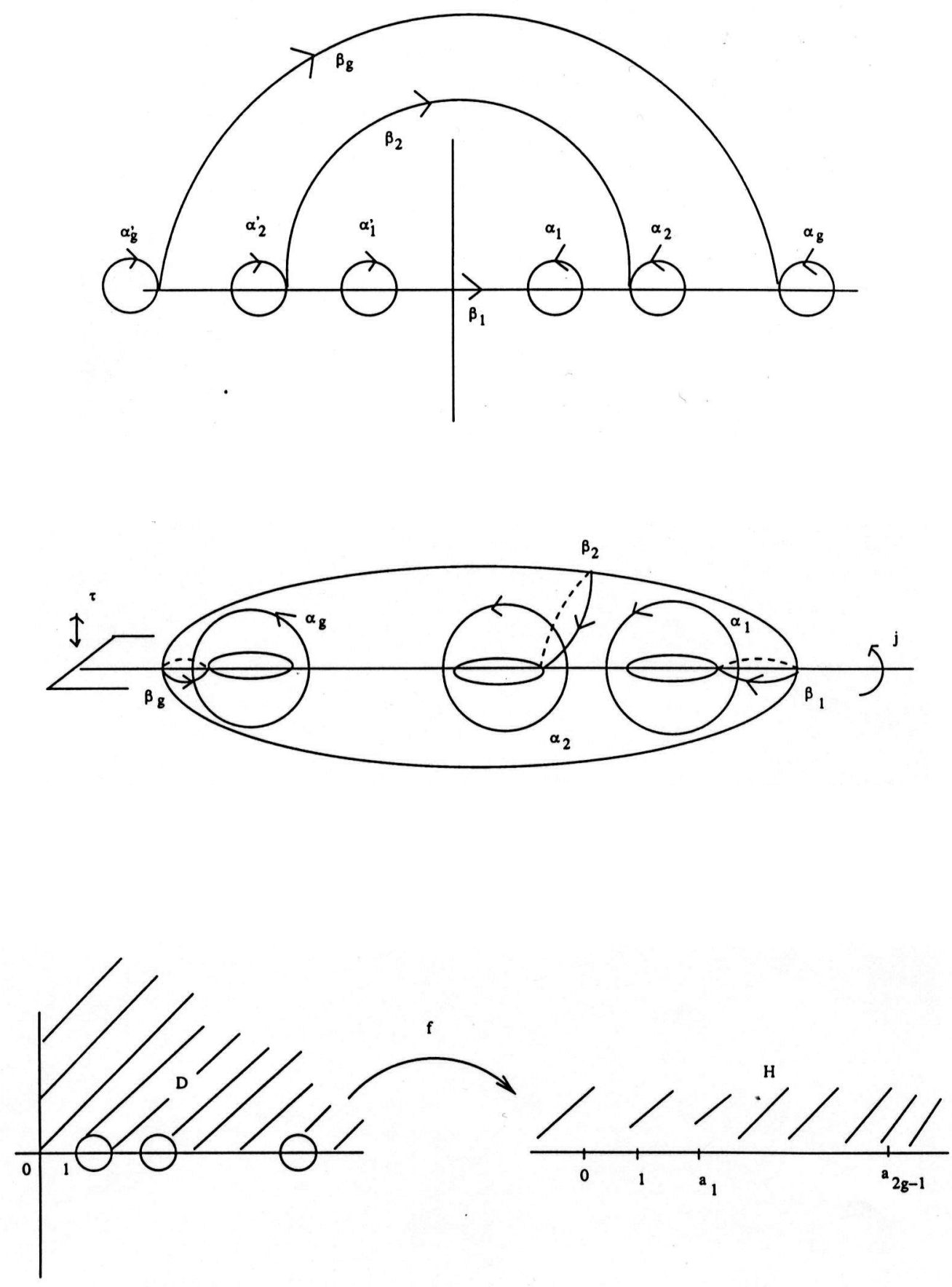


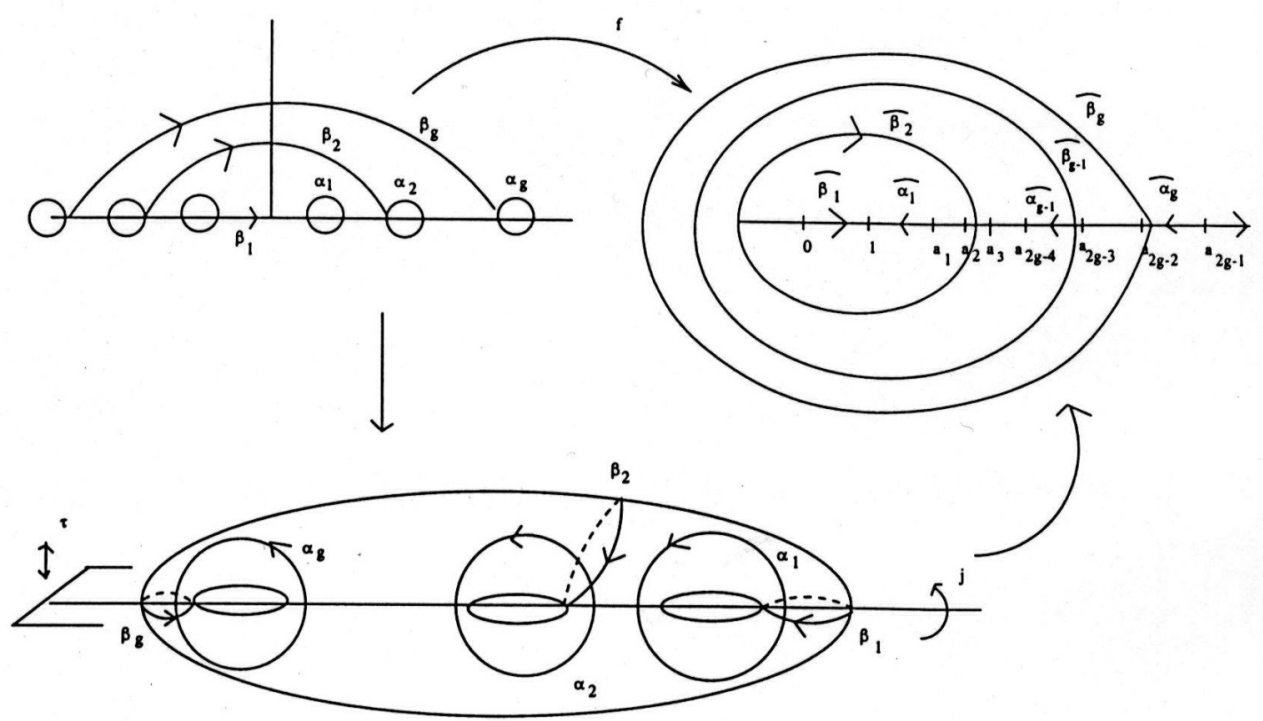

\section{References}

[1] Burnside, W. On a class of Automorphic Functions. Proc. London Math. Soc. Vol 23, pp. 49-88, (1892)

[2] Buser, P. and Silhol, R. Geodesics, periods and Equations of Real Hyperelliptic Curves. Preprint.

Received : August, 2001

\section{Rubén Hidalgo}

Departamento de Matemáticas

Universidad Técnica Federico Santa María

Casilla

Valparaíso

Chile

e-mail : rhidalgo@mat.utfsm.cl 